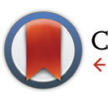

CrossMark \&lick for updates

Cite this: Polym. Chem., 2015, 6 , 2488

Received 15th December 2014, Accepted 23rd January 2015

DOI: $10.1039 / c 4 p y 01739 k$

www.rsc.org/polymers

\title{
Thermal effects in polymerisations - a live view differentiating between bulk effects, thermal diffusion, and oxygen inhibition
}

\author{
Roman Geier, ${ }^{a}$ Christina Wappl, ${ }^{a}$ Hilde Freiszmuth, ${ }^{a}$ Christian Slugovc ${ }^{\mathrm{b}}$ and \\ Georg Gescheidt*a
}

\begin{abstract}
Thermography has been shown to be an efficient tool for the screening of the efficiency of exothermic reactions. Here we show that the use of a thermal IR camera reveals effects of heat transfer in polymerising mixtures if appropriately designed reaction vessels are used. We report on case studies illustrated by photo-induced radical polymerisation of butyl acrylate and thermally triggered ring-opening metathesis polymerisation of dicyclopentadiene.
\end{abstract}

\section{Introduction}

Generally, polymerisation reactions are exothermic. It is well established that bulk effects lead to rather high temperatures in polymerising mixtures. These thermal effects have been investigated by a variety of methods, in particular DSC or in situ with thermal sensors. Another important aspect in real systems is that heat is dissipated at the interfaces between the reaction mixture and its environment, i.e., the vessel and the atmosphere. This obviously causes a rather inhomogeneous heat distribution, which may substantially alter the homogeneity and the properties of the final polymeric product. This feature is well established and has been addressed particularly using simulations. ${ }^{1-4} \mathrm{~A}$ related aspect is the inhibition of radical polymerisations by oxygen, which also appears at the interface between the polymerising formulation and air. ${ }^{5} \mathrm{~A}$ detailed analysis of these above-mentioned phenomena requires a corresponding technique, which provides information offering spatial and time resolution appropriate for polymerisation reactions.

It has been shown that the use of a thermal sensor ${ }^{6}$ and, particularly, a thermal camera provides valuable insights for assessing the efficiency of (preferably) exothermic events. ${ }^{7-9}$ Although thermal images exclusively display the temperature at the surface of the sample and disturbing reflections may occur, an image representing a two-dimensional spatial heat distribution offers useful insights into environmental effects of chemical reactions. ${ }^{10}$

\footnotetext{
${ }^{a}$ Institute of Physical and Theoretical Chemistry, Graz University of Technology, NAWI Graz, Stremayrgasse 9, 8010 Graz, Austria

${ }^{b}$ Institute for Chemistry and Technology of Materials, Graz University of Technology, NAWI Graz, Stremayrgasse 9, 8010 Graz, Austria.

E-mail: g.gescheidt-demner@tugraz.at
}

We have, therefore, evaluated the use of a high-resolution thermal camera for simultaneously following the temperature and its dissipation depending on various reaction conditions. Moreover we have developed and tested reaction vessels for this type of study. Here we report our initial results, which indicate the scope (and caveats) of this experimental approach.

\section{Results and discussion}

As a first example, we have chosen a photo-initiated radical polymerisation for our investigations. ${ }^{11-14}$ Such a procedure provides a clearly defined trigger for starting the polymerisation. In a thin-walled NMR tube, a mixture of the phosphine oxide based photoinitiator Irgacure 819 and butyl acrylate (Fig. 1A) in benzene was irradiated using a $\mathrm{Hg} / \mathrm{Xe}$ highpressure lamp for $10 \mathrm{~s}$ (shutter). The corresponding setup is shown in Fig. 1B. The $\mathrm{Hg} / \mathrm{Xe}$ lamp is oriented perpendicular toward the NMR tube. This allows following the development of heat in a cross section below and above the centre of the irradiating light. The dissipation of heat together with the corresponding thermographic curves is displayed in Fig. 1C and D. Fig. 1C shows that a hot spot is immediately created at the position where the sample is irradiated. The temperature gradually decreases at larger distances. Upon stopping irradiation after $10 \mathrm{~s}$, the temperature rises to its maximum, then gradually cools down but still being above room temperature after $140 \mathrm{~s}$. In Fig. 1D, the influence of oxygen is illustrated. The three samples shown contain identical mixtures of the initiator and butyl acrylate. The sample on the left side is degassed on a vacuum line and sealed under $\mathrm{N}_{2}$ (identical to the sample in the middle of Fig. 1C), the one in the middle reflects atmospheric conditions, whereas that on the right is saturated with $\mathrm{O}_{2}$. All three images are obtained immediately 
A)

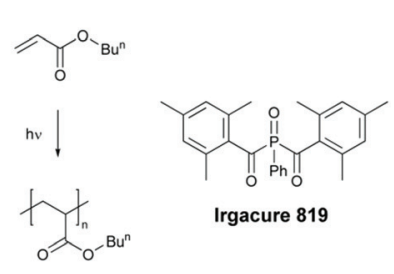

B)

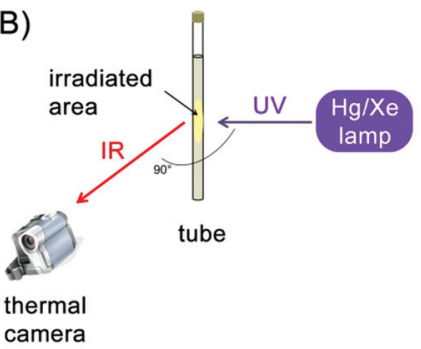

C)
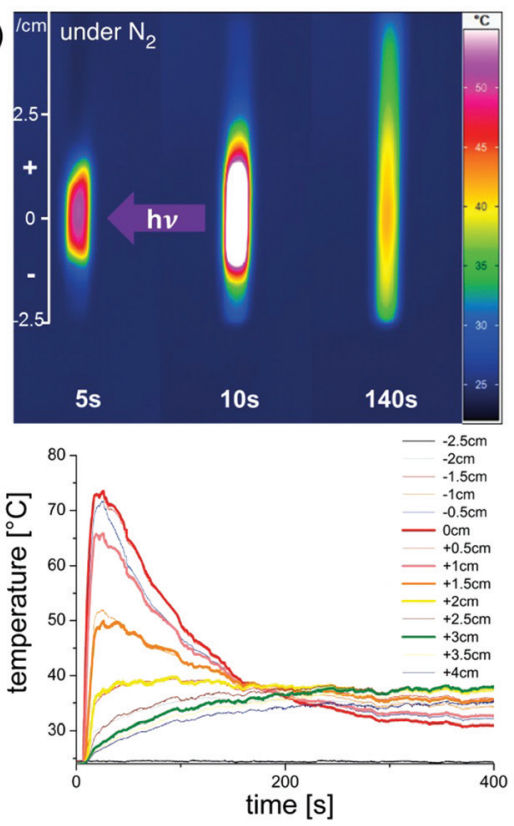
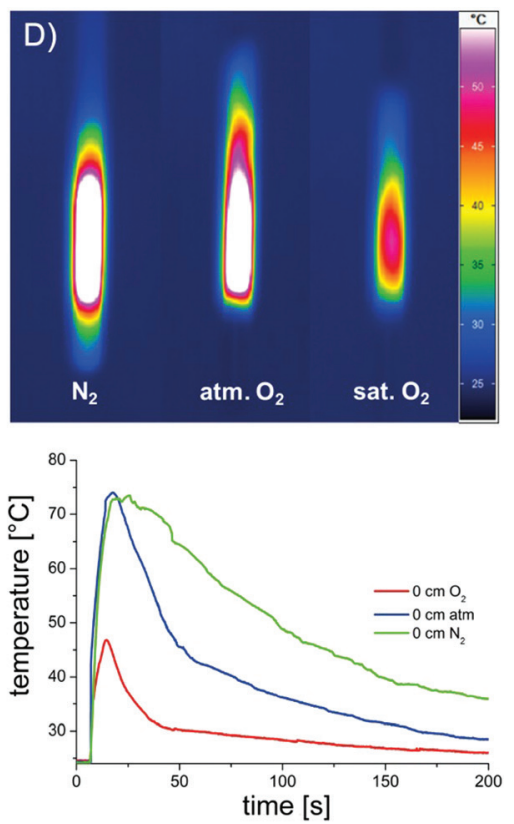

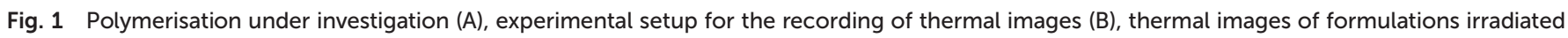

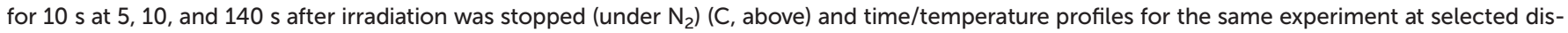

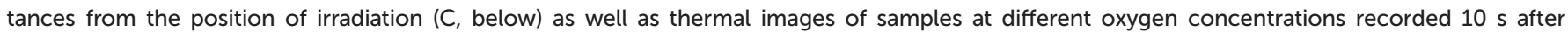
irradiation was stopped (D, above) and time/temperature profiles at the centre of irradiation of these samples (D, below).

after the irradiation ended. In the sample under an inert atmosphere, the heat is evenly distributed above and below the centre of irradiation. Although the highest temperature is essentially identical in the image in the middle, the heat distribution becomes inhomogeneous. The substantial inhibiting effect of oxygen can be clearly distinguished in the right sample: the temperature is much lower (less than half compared to the degassed case), clearly showing a substantially hampered polymerisation. This is shown in Fig. 1D (lower part) comparing the rise and decrease of the temperature in these three samples over $200 \mathrm{~s}$ at the centre of the irradiation. Whereas the green curve recorded under inert conditions $\left(\mathrm{N}_{2}\right.$ saturated sample) reaches the highest temperature $\left(T_{\max }\right)$ and decreases only slowly indicating a rather long period of polymerisation, the blue curve recorded under atmospheric atmosphere has an identical $T_{\max }$ but a substantially faster decay. When the solution is saturated with oxygen, $T_{\max }$ is markedly lower and the decay is even more pronounced. Fig. 1C reveals that particularly at later stages of the polymerisation, the dissipation of heat is not uniform, but systematically depends on convection and, possibly on the thermal properties of the environment. This is mirrored by the fact that the temperature above the centre of irradiation (positive values in Fig. 1C) is higher than that at the corresponding distance below.

The spatially-resolved images shown in Fig. 1C reveal that under an inert atmosphere, a maximum number of active initiating radicals is produced. Accordingly many polymer chains start to grow. Moreover, since chain growth is not quenched by oxygen, recombination and disproportionation reactions appear as side reactions of the radical-chain reaction. A sub- stantial portion of the heat is produced by the formation of novel $\mathrm{C}-\mathrm{C}$ bonds. As soon as oxygen is present, the formation of active initiating radicals is hindered by the deactivation of the initiating radicals by oxygen. Since the photo-induced $\alpha$ cleavage is rather fast and the quenching of the initiating radicals by $\mathrm{O}_{2}$ is a second-order diffusion-controlled reaction, this reaction is not dominating in the very first phase of polymerisation under atmospheric conditions. However, this becomes much more pronounced in the $\mathrm{O}_{2}$ saturated sample where the initiating radicals are attacked by oxygen at a higher rate. The oxygen quenching of the C-centred radicals of the growing chain is evident in both oxygen-containing samples showing a much faster decrease of the temperature relative to the degassed one.

Not all effects discussed above are exclusively caused by the role of oxygen. Heat transfer to the interfaces between the sample and the glass walls and the atmosphere definitely contribute to the efficacy of the polymerisation reaction. We have therefore specifically addressed the influence of the bulk and the interface area in a second series of experiments. To this end, we have chosen ROMP (ring-opening metathesis polymerisation, Fig. 2) since the progress of ROMP (initiated with the particular class of ruthenium compounds used) is hardly affected by the presence of oxygen and starts after an induction period of a few minutes allowing a proper mixing of the solutions and placing them in appropriate vessels. ${ }^{15}$

In a first set of measurements, four vials were filled with differing volumes of the same polymerising mixture (0.5-3.7 $\mathrm{mL}$ ) and immediately, the thermal development of the samples was monitored with the thermal camera (Fig. 2). After an induction period of $c a .4 \mathrm{~min}$, the sample with the highest 

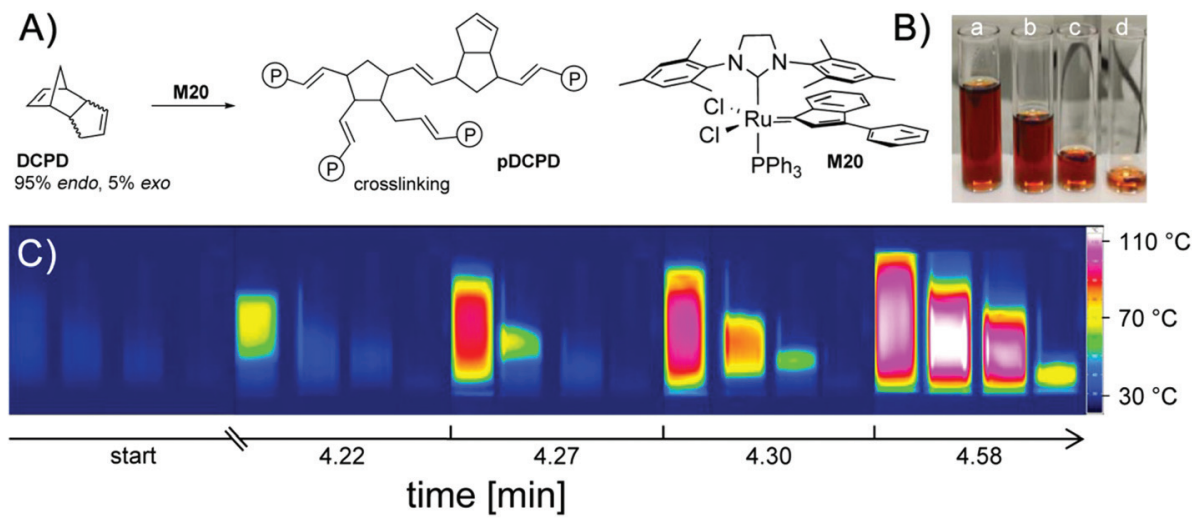

Fig. 2 ROMP of dicyclopentadiene initiated with M20 (A), photographs of reaction vessels ((a) 3.7, (b) 2.6, (c) 1.2 and (d) $0.5 \mathrm{~mL})$ ) containing the formulation for ROMP (B) and thermal images showing the heat evolvement during polymerisation depending on the volume (C).

volume starts to polymerise, consecutively followed by the samples with decreasing volumes of the formulation. The starting area of the reaction is always the (a priori) warmest region of the samples. This can be additionally perceived by the fact that even a slight heat transfer from one sample to another (if they are in close vicinity) creates a "hot spot" becoming the starting area for the polymerisation in the adjacent vessel. The lower the volume of the sample, the longer it takes for the start of the polymerisation and the lower the highest temperature achieved. Clearly these effects can be traced back to bulk effects counterbalanced by heat transfer across interfaces. To assess the influence of the overall volume and the interface area in a systematic way (walls of the sample vessel and atmosphere), we have constructed reaction chambers (Teflon) providing specific volume/interface area ratios. To avoid heat transfer between the samples, the reaction chambers were positioned well apart from each other (Fig. 3A and B). The measurement of the reaction temperature at the surface of the samples was achieved by positioning the camera above the reaction chambers. Selected results are displayed in Fig. 3C. In analogy with the measurements shown in Fig. 2, an induction period of $c a .3$ min was observed. Polymerisation started first in the sample with the highest volume. This can be seen in Fig. 3C and D, where column a, corresponding to the reaction chamber with the highest volume (Table 1), reveals the first detection of heat after $211 \mathrm{~s}$.
A)

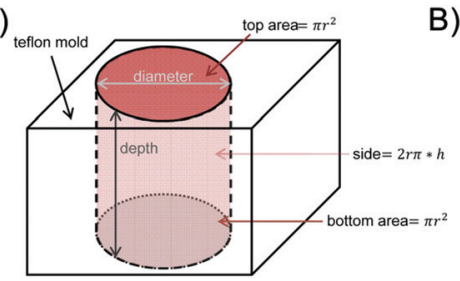

C)

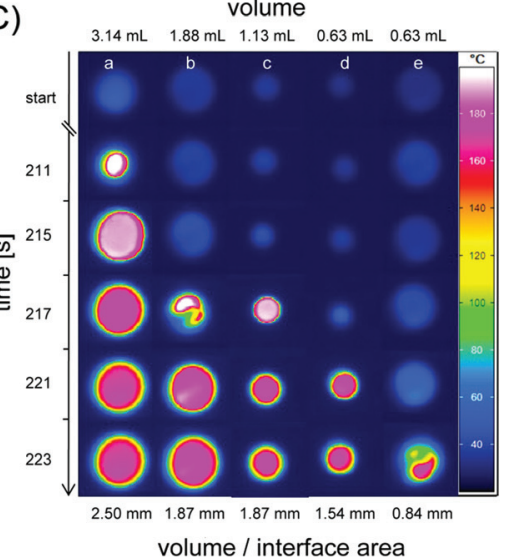

B)

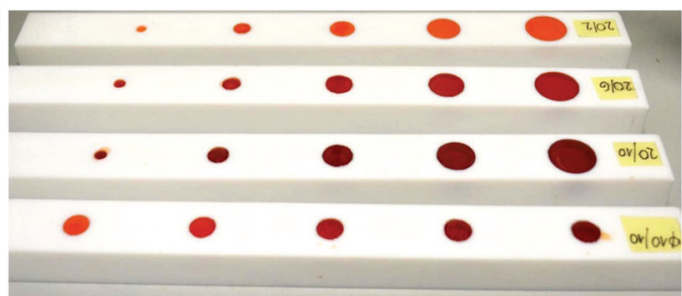

D) 225

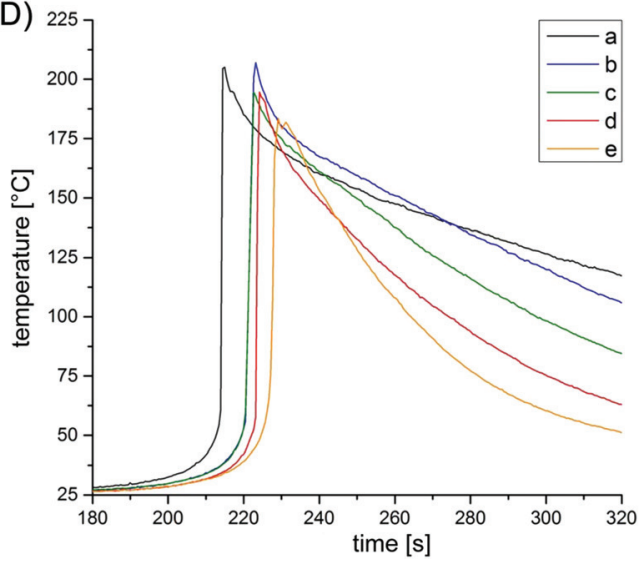

Fig. 3 General shape of the reaction chambers (A), photograph of the reaction chambers made of Teflon (B), thermal image showing the heat evolvement during ROMP in dependence of the dimensions of the reaction chambers (cf. Table 1) (C) and time/temperature profiles of the reaction chambers a-e (D). 
Table 1 Dimensions of reaction chambers

\begin{tabular}{llllll}
\hline Reaction chamber & $\mathrm{a}$ & $\mathrm{b}$ & $\mathrm{c}$ & $\mathrm{d}$ & $\mathrm{e}$ \\
\hline Diameter [mm] & 20 & 20 & 12 & 10 & 20 \\
Depth [mm] & 10 & 6 & 10 & 8 & 2 \\
Volume [mL] & 3.14 & 1.88 & 1.13 & 0.63 & 0.63 \\
Volume-interface area ratio [mm] & 2.50 & 1.87 & 1.87 & 1.54 & 0.84
\end{tabular}

Columns b-d in Fig. 3C then follow in the order of their gradually smaller volumes.

In addition to this 'volume effect', also the volume/interface ratio appears to play a major role. The polymerisation in chambers b and c starts almost simultaneously (see $4^{\text {th }}$ line in Fig. $3 \mathrm{C}$ ) although the volume of chamber $\mathrm{c}$ is only $60 \%$ of that of b. Remarkably, b and c have the same volume/interface area ratio. This implies that the heat is evenly dissipated across the interfaces between the sample and Teflon/atmosphere. Comparing the reaction in chambers $\mathrm{d}$ and e underpins the influence of the volume/interface area ratio. These chambers have identical volumes $(0.63 \mathrm{~mL})$ but different interface areas (volume/interface area ratio 1.54 and $0.84 \mathrm{~mm}$, respectively). Significantly, in sample e with a lower volume/interface area ratio, the polymerisation starts later and reaches a lower maximum temperature (Fig. 3D). Here, a larger amount of the reaction heat is transferred from the bulk to the surrounding. Generally Fig. 3D indicates that the samples with higher volume and bigger volume/interface ratio polymerise earlier and reach higher temperatures. Moreover the observation that the decay of the maximum temperature is substantially less pronounced in a, b than in d, e (Fig. 3D) is in line with the higher volume/surface ratio of the former samples.

\section{Experimental section}

For the temperature measurements, we have used an InfraTec VarioCam hr M83072 thermography system providing a $640 \times$ $480 \mathrm{dpi}$ resolution at a frequency of $60 \mathrm{~Hz}$. We used $10 \mathrm{~Hz}$ and $2 \mathrm{~Hz}$ for photoinitiated and ring opening metathesis polymerisation reactions, respectively.

Since thermal camera images exclusively present surface temperatures, our measurements were either performed in thin-walled glass tubes (NMR) or in specifically produced reaction vessels (Teflon). The specific shapes of the latter were designed to account for the effects of the overall reaction volume and the ratio of volume $v s$. the interfacing area with the atmosphere and the contact area of the polymerising mixture with the Teflon surface.

For the photoinitiated reactions benzene solutions of butyl acrylate and Irgacure 819 (phenylbisacylphosphine oxide, BASF, Germany) were used. For the measurements $0.8 \mathrm{~mL}$ of a prepared stock solution consisting of $9.8 \mathrm{wt} \%$ benzene, $0.1 \mathrm{wt} \%$ Irgacure 819 and $90.1 \mathrm{wt} \%$ of butyl acrylate was filled in thinwalled $5 \mathrm{~mm}$ NMR tubes. The NMR tubes were sealed with septa and either degassed ( $\mathrm{N}_{2}$ bubbling for $3 \mathrm{~min}$ ) or enriched with oxygen under light exclusion (wrapped in aluminium foil). Samples representing atmospheric oxygen levels were kept untreated. Every sample was freshly prepared before the experiment. A Hg/Xe lamp (Hamamatsu LC4, L8252 lamp, max at $365 \mathrm{~nm}$ ) equipped with a light guide (diameter $5 \mathrm{~mm}$ ) was used. This setup yielded $2200 \pm 300 \mathrm{~mW} \mathrm{~cm}{ }^{-2}$. A cross section of $15 \mathrm{~mm}$ was irradiated for $10 \mathrm{~s}$ (shutter). The recording of the thermal response was started $7 \mathrm{~s}$ before irradiation.

For the ring opening metathesis polymerisation in bulk, dicyclopentadiene $(97 \%$, a mixture of endo and exo isomer (95:5), ABCR; $7.84 \mathrm{~g}$ and $14.7 \mathrm{~g}$ for measurements in glass vials and Teflon moulds, respectively) was molten and mixed with dichloromethane ( $30 \mu \mathrm{L}$ per $\mathrm{mL}$ DCPD) to keep it in the liquid state. A stock solution of the initiator M20 (Umicore) ${ }^{16}$ in dichloromethane $\left(34.5 \mathrm{mg} \mathrm{mL} \mathrm{m}^{-1}\right)$ was prepared. For the thermal measurements, the polymerisation was initiated by the addition of initiator-solution $(240$ and $450 \mu \mathrm{L}$; equal to $150 \mathrm{ppm} \mathrm{M20}$ in respect of DCPD) to the monomer. Subsequently, the formulation was filled in the respective moulds and the heat evolvement was measured.

\section{Conclusions}

Although thermal measurements were reported for several cases as a (semi)quantitative tool for describing the efficiency of chemical reactions, ${ }^{17}$ our results illustrate pretty well the power of thermography to visualize, study and quantify the polymerisation progress under different conditions. The aim of our investigation was testing whether the use of a thermal camera provides new and useful insights into polymerisation phenomena. The images and the curves shown in Fig. 1 and 3 illustrate that we have been capable of systematically establishing and distinguishing bulk effects, heat transfer, the influence of interfaces, and the impact of oxygen. Although the recorded images provide only information on the temperature of the surface of the investigated samples, the non-isotopic heat flow can be followed rather precisely and reproducibly.

In these terms, the use of a high-resolution thermal camera is very useful for investigating the efficiency of the formation of polymers on a convenient time scale ( $1 / 60 \mathrm{~s})$ and an appropriate resolution (sub $\mathrm{mm}$ ), which is likely to be useful for studying rather subtle details during the curing of photo-polymerisable coatings in addition to standard IR procedures monitoring the conversion of double bonds. ${ }^{18}$ Such investigations require custom-made reaction chambers that allow following the desired phenomena, always bearing in mind that the surface temperature is monitored exclusively. It is also crucial to control the environmental conditions very precisely to achieve a perfect reproducibility of the results.

We are currently expanding our studies toward frontal polymerisation, non-homogeneous systems, etc. and developing specific vials for enhancing the scope of this approach. A detailed look on thermographic images seems to be valuable and should also enhance the development of theoretical models. ${ }^{19}$ 


\section{Acknowledgements}

We are indebted to Herbert Lang (Workshop Physical and Theoretical Chemistry TU Graz) for preparing the Teflon-based reaction vessels.

\section{Notes and references}

1 G. L. Batch and C. W. Macosko, J. Appl. Polym. Sci., 1992, 44, 1711-1729.

2 A. Boddapati, S. B. Rahane, R. P. Slopek, V. Breedveld, C. L. Henderson and M. A. Grover, Polymer, 2011, 52, 866873.

3 C. E. Corcione, A. Greco and A. Maffezzoli, Polym. Eng. Sci., 2006, 46, 493-502.

4 P. M. Johnson, J. W. Stansbury and C. N. Bowman, Macromolecules, 2008, 41, 230-237.

5 S. C. Ligon, B. Husar, H. Wutzel, R. Holman and R. Liska, Chem. Rev., 2014, 114, 557-589.

6 B. J. Falk, S. M. Vallinas and J. V. Crivello, J. Polym. Sci., Part A: Polym. Chem., 2003, 41, 579-596.

7 A. Holzwarth, H.-W. Schmidt and W. F. Maier, Angew. Chem., Int. Ed., 1998, 37, 2644-2647.

8 M. T. Reetz, M. H. Becker, K. M. Kühling and A. Holzwarth, Angew. Chem., Int. Ed., 1998, 37, 2647-2649.
9 Y. M. Kim, L. K. Kostanski, J. F. MacGregor and A. E. Hamielec, J. Therm. Anal. Calorim., 2004, 78, 153-164.

10 T. Nagasawa, B. Ochiai and T. Endo, J. Polym. Sci., Part A: Polym. Chem., 2006, 44, 5519-5524.

11 K. Dietliker, T. Jung, J. Benkhoff, H. Kura, A. Matsumoto, H. Oka, D. Hristova, G. Gescheidt and G. Rist, Macromol. Symp., 2004, 217, 77-97.

12 I. Gatlik, P. Rzadek, G. Gescheidt, G. Rist, B. Hellrung, J. Wirz, K. Dietliker, G. Hug, M. Kunz and J.-P. Wolf, J. Am. Chem. Soc., 1999, 121, 8332-8336.

13 S. Jockusch and N. J. Turro, J. Am. Chem. Soc., 1998, 120, 11773-11777.

14 Y. Yagci, S. Jockusch and N. J. Turro, Macromolecules, 2010, 43, 6245-6260.

15 A. Leitgeb, J. Wappel, C. A. Urbina-Blanco, S. Strasser, C. Wappl, C. S. J. Cazin and C. Slugove, Monatsh. Chem., 2014, 145, 1513-1517.

16 J. Broggi, C. A. Urbina-Blanco, H. Clavier, A. Leitgeb, C. Slugovc, A. M. Z. Slawin and S. P. Nolan, Chem. - Eur. J., 2010, 16, 9215-9225.

17 J. Loskyll, K. Stoewe and W. F. Maier, ACS Comb. Sci., 2012, 14, 295-303.

18 K. Taki, Y. Watanabe, H. Ito and M. Ohshima, Macromolecules, 2014, 47, 1906-1913.

19 M. D. Goodner and C. N. Bowman, Chem. Eng. Sci., 2002, 57, 887-900. 